(2)

OPEN ACCESS

\title{
Efficacy of patent foramen ovale closure for treating migraine: a prospective follow-up study
}

\author{
Yi Qi (1) , 'Yushun Zhang, ${ }^{2}$ Xiaohui Luo, ${ }^{2}$ Gesheng Cheng, ${ }^{2}$ Yajuan Du, ${ }^{2}$ Rui Liu, ${ }^{1}$ \\ Hui Xie, ${ }^{1}$ Yawen Cheng, ${ }^{1}$ Yichen Guo (1) , ${ }^{1}$ Guogang Luo ${ }^{1}$
}

${ }^{1}$ Department of Neurology, Xi'an Jiaotong University Medical College First Affiliated Hospital, Xi'an, Shaanxi, China

${ }^{2}$ Department of Structural Heart Disease, Xi'an Jiaotong University Medical College First Affiliated Hospital, Xi'an, Shaanxi, China

\section{Correspondence to} Professor Guogang Luo, Department of Neurology, Xi'an Jiaotong University Medical College First Affiliated Hospital, Xi'an, Shaanxi, China; lguogang@163.com

Accepted 4 August 2020 Published Online First 14 September 2020
D) Check for updates

(C) American Federation for Medical Research 2021. Re-use permitted under CC BY-NC. No commercial re-use. Published by BMJ.

To cite: Qi Y, Zhang Y, Luo $\mathrm{X}$, et al. J Investig Med 2021;69:7-12.

\section{ABSTRACT}

This study aims to evaluate the potential of percutaneous patent foramen ovale (PFO) closure to improve the headache in patients with migraine and PFO, and discuss the difference between the randomized controlled trials (RCTs) and the singlecenter studies. Patients of migraine with a large shunt of PFO, who experienced $\geq 2$ headache attacks per month and failed $\geq 2$ categories of standardized medication, underwent PFO closure in First Affiliated Hospital of Xi'an Jiao Tong University. The clinical outcomes, including frequency and duration of headache attacks, Headache Impact Test (HIT-6) score, and Visual Analogue Scale (VAS) score, were evaluated at 3,6 , and 12 months of follow-up after the PFO closure. The different efficacies of the clinical outcomes between patients with and without aura as well as different grades of PFO were also evaluated, respectively. 134 patients with migraine (39 male and 95 female) with PFO were enrolled, whose average age was $39.21 \pm 11.37$ years. After PFO closure, there was a significant reduction in frequency and duration of headache attacks, HIT-6 score, and VAS score at 3, 6, and 12 months' followup $(p<0.001)$. Migraine was completely relieved in 54 (40.30\%) patients during 12 months' follow-up. The frequency of migraine was reduced by $>50 \%$ in $44(32.84 \%)$ patients at 3 months' follow-up and increased to $48(35.82 \%)$ at 12 months' follow-up. $31.03 \%$ patients remained residual shunt after 6 months of closure with varying improvements of headache. This study confirmed that PFO closure can effectively reduce frequency and duration of migraine and improve quality of life, but the definitive indications and long-term effect still need further research.

\section{INTRODUCTION}

Migraine is a common, chronic, and neurovascular disorder characterized by self-limited, recurrent moderate-to-severe headaches associated with autonomic symptoms. Also, 14.7\% of global migraine prevalence poses a heavy burden on public health. ${ }^{1}$ Patent foramen ovale (PFO) refers to the remnant opening of the fetal foramen ovale, which is a connection between the left and right atrium. A prospective, population-based study has revealed that the incidence of PFO could reach up to $25.6 \%$ by esophageal echocardiography. ${ }^{2}$ The prevalence

\section{Significance of this study}

What is already known about this subject?

- Migraine is highly correlated to patent foramen ovale (PFO), especially migraine with aura.

- Up to date, 3 large randomized controlled trials (RCTs) did not reach the end points, but most single-center observations showed that PFO closure can effectively prevent migraine attack.

- The potential and the definitive indications, and long-term effect of PFO closure to improve the headache attacks in migraine with PFO are still unclear.

\section{What are the new findings?}

- This one year follow-up study confirmed that PFO closure can effectively reduce frequency and duration of migraine and improve quality of life.

- The different inclusion criteria, primaryl secondary endpoints, and follow-up times may be the causes of single-center observations different from the RCTs' results. The residual shunt may still cause headache attacks after PFO closure.

- Antiplatelet aggregation drugs like clopidogrel may be an effective prophylactic for patients of migraine with PFO.

How might these results change the focus of research or clinical practice?

- There will be definitive indications to treat patients with migraine and PFO clinically. More patients with migraine can get relief from headache attacks.

of PFO was $20 \%-30 \%$ in the general population and $27 \%$ of in autopsy. ${ }^{3}$ The correlation between PFO and migraine was originally reported in a case-control study conducted by Del Sette et al in 1998. ${ }^{4}$ Subsequently, other scholars reported similar conclusions. A metaanalysis conducted by Schwedt et al in 2008 shows that the prevalence of PFO in patients with migraine ranged from $39.8 \%$ to $72 \%$, and the prevalence of migraine in subjects with $\mathrm{PFO}$ also fluctuated from $22.3 \%$ to $64.3 \% .^{5}$ 
Although the International Headache Society and the Neurological Society do not recommend percutaneous PFO closure as a routine treatment for patients with migraine, there is no doubt that migraine, especially migraine with aura (MA), is highly correlated to PFO. ${ }^{6-8}$ PFO closure has been one of the potentially effective treatments to prevent headache attacks in patients with migraine for decades. Up to date, most single-center observations showed that PFO closure can effectively prevent migraine attack, but three large randomized controlled trials (RCTs), MIST, ${ }^{9}$ PRIMA, ${ }^{10}$ and PREMIUM, ${ }^{11}$ have all shown negative results. Therefore, the therapeutic effects of this surgical procedure remain controversial. ${ }^{9-11}$ What causes the difference between single-center observation results and large-scale RCTs' results is highly worthy to elucidate. The primary aim is to evaluate the efficacy of percutaneous PFO closure in patients with migraine with PFO in this singlecenter study. Second, we also try to discuss the possible causes for the different results between RCTs and singlecenter studies.

\section{MATERIALS AND METHODS Patients}

Migraine was diagnosed according to the third edition of the International Classification of Headache Disorders (ICHD-III). ${ }^{5}$ All patients with migraine were diagnosed and enrolled from the Headache Clinic and the Department of Neurology, the First Affiliated Hospital of Xi'an Jiao Tong University. A written informed consent was obtained from each participant.

The main inclusion criteria consist of patients aged 16-70 who can withstand and agree to the PFO closure, $\geq 2$ migraine headache attacks per month and at least half a year of migraine course, failed $\geq 2$ categories of standardized migraine medication, and presented with a large shunt of PFO after the Valsalva maneuver by contrast transthoracic echocardiography (cTTE).

Exclusion criteria included other types of headache or cardiovascular defects, other neurological disorders, pregnancy or lactation, any other medical condition or contraindication to the procedures and treatments used in the study. The present study was approved by the local Ethics Committee(KYLLSL-2013-007-01).

\section{Identification of PFO}

PFO was identified using a multi-modality screening method, which included cTTE, transesophageal echocardiography, and contrast transcranial Doppler. The PFO was classified into four grades according to the cTTE at the resting state: (1) grade 0 , no shunt (no bubble); (2) grade 1, small shunt (1-10 bubbles); (3) grade 2, middle shunt (11-30 bubbles); (4) grade 3, large shunt ( $>30$ bubbles or full of bubbles in the left atrium). As previously reported, CTTE is a non-invasive, convenient, and economical modality that can improve the diagnostic sensitivity of PFO to as high as $63 \%-100 \% .{ }^{12}$ All the patients in this study who were identified with large PFO by cTTE presented with a large shunt after the Valsalva maneuver and underwent percutaneous PFO closure in the Department of Structural Heart Disease.

\section{Percutaneous PFO closure}

Routine preoperative examinations were performed, such as ECG, blood routine, and coagulant function. A 6F catheter was implanted through the right femoral vein and advanced up to the right atrium. Heparin (4000 IU) was administered, and pulmonary artery pressure and right ventricular pressure were measured. The sizes of the Amplatzer Occluder and Cardi-O-Fix Occluder were determined according to the in vitro measurement of the balloon, the body size and weight et all. At the same time, the cTTE showed that the occluder was fixed in position and in good shape. The catheter was pulled back and the occluder was fully released without residual shunt. All operations are performed by experienced surgeons in the Department of Structural Heart Disease. Aspirin (100 mg/day) was used for 6 months, and clopidogrel $(75 \mathrm{mg} /$ day $)$ was used for 3 months after PFO closure. All patients were allowed to use other acutephase treatments.

\section{Outcomes and follow-up}

The clinical outcomes, including the frequency and duration of headache attacks, the Headache Impact test (HIT-6) score, and the Visual Analogue Scale (VAS) score, were mainly evaluated.

The primary efficacy end point was the improvement of headache frequency and duration after PFO closure. The headache frequency included the headache attacks that meet migraine diagnostic criteria by ICHD-III or headache duration that does not meet diagnostic criteria but patient takes acute rescue analgesic. The duration was counted by hours which meet the characteristics of a migraine attack with or without acute medication. The headache frequency and duration was calculated by monthly average during the follow-up period. Secondary end points of efficacy were (1) the change in the severity of migraine attacks based on HIT-6 score and VAS score, (2) headache characteristics and the efficacy of PFO closure between patient with and without aura, and (3) headache characteristics and the efficacy of PFO closure among four grades of PFO.

Follow-up data for all patients were obtained at clinic visit or via telephone interviews at 3,6, and 12 months after PFO closure, respectively.

\section{Statistical analysis}

SPSS V.24.0 software (IBM) was used for statistical analysis. Continuous data were expressed as mean $\pm S D$, and compared using Student's t-test or Mann-Whitney U test. Quantitative data were described as frequencies and/ or percentages, and these were compared using $\chi^{2}$ test. Probability (p) values $\leq 0.05$ were considered statistically significant.

\section{RESULTS}

\section{Demographic and clinical characteristics}

A total of 134 patients with migraine with PFO were successfully enrolled. The demographic and clinical characteristics are summarized in table 1 . There were 39 (29.10\%) male and $95(70.90 \%)$ female patients, and their average age was $39.21 \pm 11.37$ years (range, $16-70$ years). The mean course of migraine was $7.74 \pm 6.84$ years. 
Table 1 Demographic and clinical characteristics of migraineurs with PFO

\begin{tabular}{ll}
\hline Characteristics & Number \\
\hline Gender (female) & $95(70.90 \%)$ \\
\hline Age (years) & $39.21 \pm 11.37$ \\
\hline Height (cm) & $164.93 \pm 6.39$ \\
\hline Weight (kg) & $62.71 \pm 10.89$ \\
\hline BMI (kg/m ${ }^{2}$ ) & $22.98 \pm 3.30$ \\
\hline Hypertension, $\mathrm{n}(\%)$ & $15(11.19)$ \\
\hline Coronary heart disease, $\mathrm{n}(\%)$ & $2(1.49)$ \\
\hline Diabetes, $\mathrm{n}(\%)$ & $1(0.75)$ \\
\hline Hyperlipidemia, $\mathrm{n}(\%)$ & $3(2.24)$ \\
\hline Smoking, $\mathrm{n}(\%)$ & $22(16.42)$ \\
\hline ASA, $\mathrm{n}(\%)$ & $10(7.46)$ \\
\hline ASD, $\mathrm{n}$ (\%) & $4(2.99)$ \\
\hline ASA with ASD, $\mathrm{n}(\%)$ & $1(0.75)$ \\
\hline Migraine characteristics & \\
\hline Onset age (years) & $33.25 \pm 12.94$ \\
\hline Course (years) & $7.74 \pm 6.84$ \\
\hline Migraine with aura, $\mathrm{n}(\%)$ & $43(32.09)$ \\
\hline Migraine without aura, $\mathrm{n}(\%)$ & $91(67.90)$ \\
\hline Chronic migraine & $47(35.07)$ \\
\hline Frequency (per month) & $11.53 \pm 11.06$ \\
\hline Duration (hours) & $12.43 \pm 15.25$ \\
\hline VAS score & $4.57 \pm 2.17$ \\
\hline HIT-6 score & $61.18 \pm 7.97$ \\
\hline
\end{tabular}

ASA, atrial septal aneurysm; ASD, atrial septal defect; HIT-6, Headache Impact Test; PFO, patent foramen ovale; VAS, Visual Analogue Scale.

\section{Safety of PFO closure}

The percutaneous PFO closure was successful and the postoperative course was uneventful in all patients. There was no embolism, hematoma, dislocation, or shedding of the occluder. All patients were discharged at 2-4 days after PFO closure.

\section{Improvement of headache}

Overall, migraine was gradually improved after PFO closure over time. After a 3-month follow-up, migraine was completely relieved in $44(32.84 \%)$ patients. After 12 months, migraine was completely relieved in 54 (40.30\%) patients. Furthermore, after 3 months, 44 $(32.84 \%)$ patients had a $>50 \%$ reduction in headache attacks. After 12 months, this number increased to 48 $(35.82 \%)$. The main evaluation of clinical outcomes before and after PFO closure are listed in table 2. There was a significant reduction in the frequency and duration of headache attacks, HIT-6 score, and VAS score at 3, 6, and 12 months after PFO closure (all p $<0.001$; figure 1 ). Further analysis revealed that headache frequency and
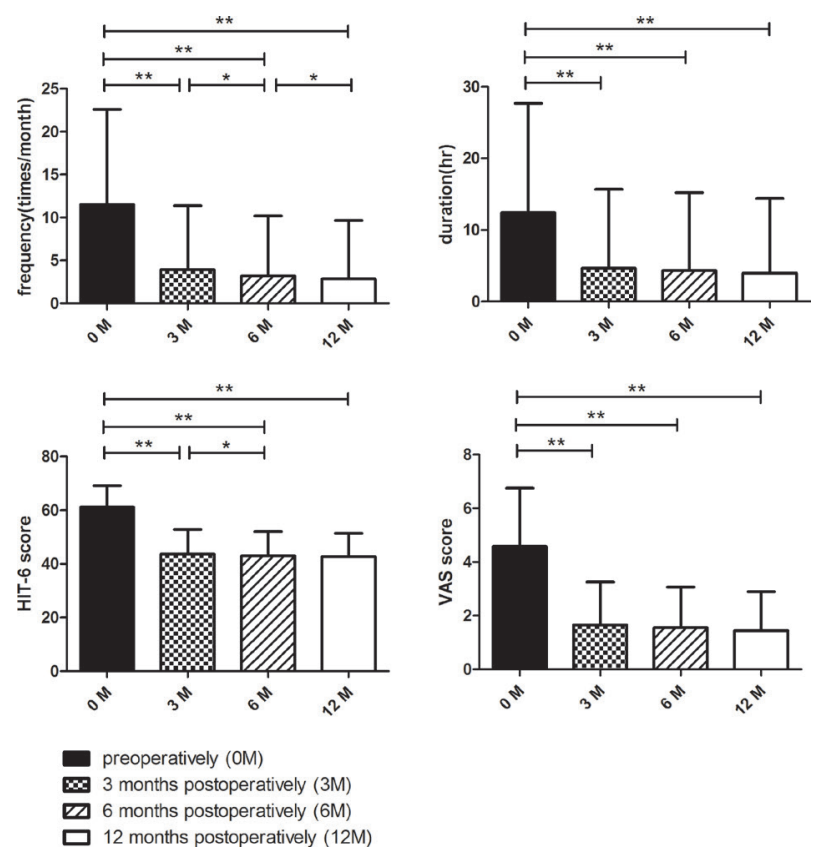

Figure 1 Headache frequency, headache duration, Headache Impact Test (HIT-6) score, and Visual Analogue Scale (VAS) score preoperatively $(0 \mathrm{M})$, at 3 months postoperatively (3M), at 6 months postoperatively $(6 \mathrm{M})$, and at 12 months postoperatively $(12 \mathrm{M})$. $p<0.001$, compared with the preoperative baseline. ${ }^{*} p<0.05$, compared with the preoperative baseline or the last follow-up time point.

HIT-6 score after the 6-month follow-up were significantly lower than those after the 3-month follow-up $(p=0.004$ and $p=0.028$, respectively). Moreover, headache frequency after the 12 -month follow-up was significantly lower than that after the 6-month follow-up $(\mathrm{p}=0.038)$.

\section{Association between aura and clinical outcomes of headache}

In the present cohort, aura was noted in 43 (32.09\%) patients. There was no significant difference in course of migraine, headache frequency, duration, or VAS score between patients with MA and patients with migraine without aura (MoA) (all p>0.05), while HIT-6 score was significantly higher in patients with MA than patients with MoA $(p=0.009)$. The data regarding MA and MoA are summarized in table 3 . Regrettably, there was no other significant difference in efficacy of PFO closure between patients with MA and MoA ( $p>0.05$ ) in our research.

Table 2 Main evaluation of clinical outcomes before and after PFO closure

\begin{tabular}{llllc}
\hline Clinical outcomes & OM & $3 \mathrm{M}$ & $6 \mathrm{M}$ & $12 \mathrm{M}$ \\
\hline Frequency (per month) & $11.53 \pm 11.06$ & $3.92 \pm 7.47$ & $3.23 \pm 6.97$ & $2.84 \pm 6.81$ \\
Duration (hours) & $12.43 \pm 15.25$ & $4.64 \pm 11.04$ & $4.34 \pm 10.88$ & $4.00 \pm 10.40$ \\
VAS score & $4.57 \pm 2.17$ & $1.66 \pm 1.60$ & $1.55 \pm 1.51$ & $1.44 \pm 1.45$ \\
HIT-6 score & $61.18 \pm 7.97$ & $43.65 \pm 9.17$ & $43.06 \pm 8.95$ & $42.70 \pm 8.76$ \\
\hline
\end{tabular}

HIT-6, Headache Impact Test; 0M, preoperatively; 3M, at 3 months postoperatively; $6 \mathrm{M}$, at 6 months postoperatively; $12 \mathrm{M}$, at 12 months postoperatively; PFO, patent foramen ovale; VAS, Visual Analogue Scale. 


\begin{tabular}{|c|c|c|c|c|}
\hline $\begin{array}{l}\text { Table } 3 \mathrm{He} \\
\text { MoA }\end{array}$ & \multicolumn{4}{|c|}{ Headache characteristics before occlusion in MA and } \\
\hline Characteristics & MA $(n=43)$ & $\operatorname{MoA}(n=91)$ & Statistics & $P$ value \\
\hline Course (years) & $8.41 \pm 7.19$ & $7.42 \pm 6.69$ & $Z=-0.992$ & 0.321 \\
\hline $\begin{array}{l}\text { Frequency (per } \\
\text { month) }\end{array}$ & $11.99 \pm 11.68$ & $11.32 \pm 10.82$ & $Z=-0.144$ & 0.886 \\
\hline Duration (hours) & $9.59 \pm 14.16$ & $13.77 \pm 15.64$ & $Z=-0.992$ & 0.076 \\
\hline VAS score & $4.77 \pm 2.10$ & $4.48 \pm 2.21$ & $Z=-0.992$ & 0.415 \\
\hline HIT-6 score & $63.79 \pm 7.43$ & $59.95 \pm 7.95$ & $t=2.668$ & 0.009 \\
\hline
\end{tabular}

HIT-6, Headache Impact Test; MA, migraine with aura; MoA, migraine without aura; VAS, Visual Analogue Scale.

\section{Abnormality in cTTE}

All patients in the present study preoperatively underwent cTTE at the resting state and after Valsalva maneuver. All patients had a large PFO presented with a large right to left shunt after the Valsalva maneuver by cTTE . According to the PFO grading system by cTTE at the resting state, 34 (25.37\%) patients were grade 0 (no shunt), 57 (42.54\%) patients were grade 1 (small shunt), 20 (14.93\%) patients were grade 2 (middle shunt), and 23 (17.16\%) patients were grade 3 (large shunt). There was no significant difference in headache frequency, headache duration, VAS score, or HIT-6 score among patients with PFO in different grades before PFO closure $(p=0.652, p=0.622, p=0.126$, $\mathrm{p}=0.267$ ). According to the follow-up data, the VAS score of patients with small shunt was significantly improved at 3 months postoperatively (figure 2). However, there was no statistical difference in the efficacy among each grade at 6 and 12 months after PFO closure. After 6 months, 87 patients were re-examined by cTTE, in which 27 patients $(31.03 \%)$ presented residual shunt. Eleven patients (40.74\%) had no headache attacks. Also, there were 10 patients $(37.04 \%)$ who had a $>50 \%$ reduction in headache attacks. However, there is indeed one patient with no significant change in headache attacks after 6 months' PFO closure with the residual shunt. There was no significant

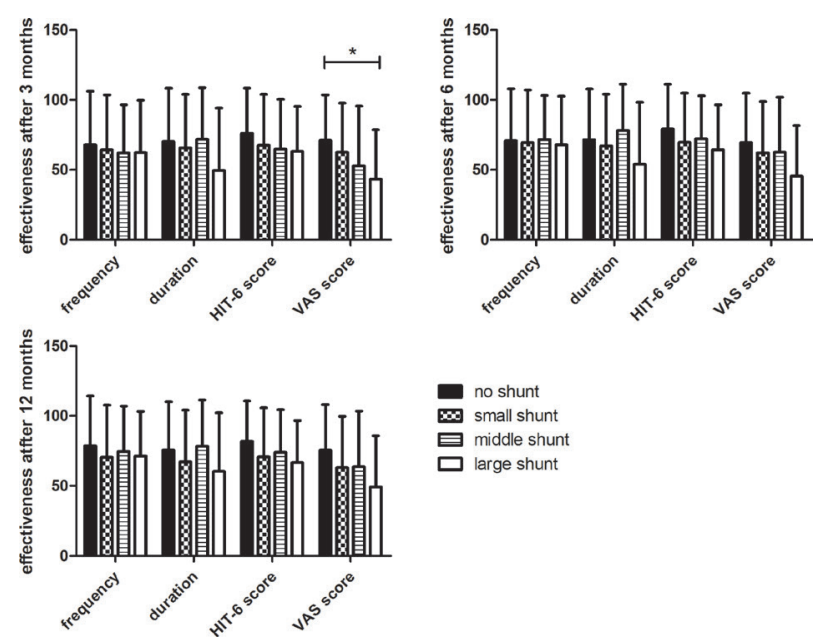

Figure 2 Effectiveness of headache frequency, duration, Headache Impact Test (HIT-6) score, and Visual Analogue Scale (VAS) score among each grade of shunt after 3 months, 6 months, and 12 months postoperatively. ${ }^{*} p<0.05$, compared with the effectiveness among each grade. difference in efficacy of PFO closure between patients with residual shunt and without residual shunt.

In addition, 10 patients had atrial septal aneurysm (ASA), 4 patients had atrial septal defect (ASD), and 1 patient had coexisting ASA and ASD. Headache duration of patients combined with PFO and ASA or ASD was 2.39 \pm 1.58 , $2.49 \pm 1.58$, and $2.66 \pm 1.57$ hours at 3,6 , and 12 months after PFO closure, which was shorter than the patients with PFO alone (4.92 $\pm 1.06,4.57 \pm 1.04,4.17 \pm 0.99, \mathrm{p}>0.05)$. The frequency of headache attacks in patients with ASA or ASD was reduced from $16.15 \pm 3.32$ per month to $3.17 \pm 1.99,3.43 \pm 2.01$, and $3.08 \pm 1.95$ per month at 3 , 6 , and 12 months, but there was no statistical difference ( $p>0.05)$ with the PFO alone. Similarly, the VAS score and HIT-6 score of patients with ASA or ASD were lower than the PFO alone, but there was no statistical difference.

\section{Influence of the course on PFO closure}

According to the ICHD-III, 47 (35.07\%) patients were diagnosed as chronic migraine. There was no significant difference in the efficacy of PFO closure between patients with chronic migraine and other patients $(p>0.05)$.

\section{DISCUSSION}

Migraine is a disabling disorder that seriously impairs an individual's quality of life. The prevalence of PFO is remarkably high in patients with migraine, cryptogenic stroke, transient ischemic attack, vertigo, and syncope. ${ }^{13-15}$ Our previous research results showed the presence of PFO was found in $56.8 \%(151 / 266)$ of all migraine patients, of whom 65 cases presented with large shunts. This is consistent with the conclusions reported by other scholars, ${ }^{16-18}$ and a meta-analysis in 2008 revealed that the frequency of PFO in migraineurs could reach up to $39.8 \%-72.0 \%$. $^{15}$

Based on possible pathogenesis of the "paradoxical embolism" theory and the cortical spreading depression theory, more and more studies try to explore the improvement of headache symptoms in patients with migraine with PFO after PFO closure. Regrettably, three RCTs reported in the literature have all shown negative results. The first was the MIST trial, which included 74 patients with MA in the PFO closure group and 73 patients with MA in the sham group. All patients had $>5$ migraine headache days per month and a history of having failed at least two classes of preventive medication. The primary end point was defined as complete cessation of migraine and the secondary end point was $\geq 50 \%$ reduction of headache days. After 6 months of follow-up, the study failed to meet its primary and secondary end points. However, the frequency of headache attacks decreased by $3.26 \pm 1.82$ days in the PFO closure group. In our study, $47(35.07 \%)$ patients with chronic migraine also did not gain more benefits from percutaneous PFO closure when compared with the others. It was speculated that these patients may have a lower pain threshold. The PRIMA trial had a total of 107 patients with migraine with $\mathrm{PFO}^{10} ; 40 \%$ were randomized to PFO closure and the rest to no sham group. Although the study failed to meet its primary end point after 12 months of follow-up, which was greater than $50 \%$ reduction in migraine days at 1 year, the intervention group has 2.9 days' reduction in total migraine days (without and with aura). Specifically, PFO closure 
yielded a reduction in migraine with aura days of 2.4 versus 0.6 in control group $(p=0.01)$, as well as migraine with aura attacks $(2.0$ vs $0.5, \mathrm{p}<0.01)$. A total of 230 subjects were enrolled in the PREMIUM study ${ }^{7}$ with 123 in the PFO closure plus medical therapy arm and 107 in the medical arm only. There was a statistically significant reduction of mean migraine days/month between intervention group and control group (3.4 vs 2.0, $\mathrm{p}=0.03$ ).

Conversely, many single centers reported that PFO closure can effectively prevent migraine attack. Morandi et al evaluated the frequency, duration, and intensity of headaches in 17 patients during the follow-up period after PFO closure, and they found that the symptoms were completely relieved in five cases and significantly improved in 10 cases. ${ }^{19}$ Other retrospective studies also demonstrated that PFO closure could significantly alleviate headache symptoms. ${ }^{20-22}$ In the present study, $40.30 \%$ patients had no headache attack at 12 months after PFO closure. Moreover, $32.84 \%$ of patients had a $>50 \%$ reduction in headache attacks at 3 months postoperatively, and this proportion increased to $35.82 \%$ at 12 months postoperatively. In fact, these findings were consistent with the results of the PREMIUM trial, in which $38 \%$ of patients had a $>50 \%$ reduction in headache attacks after PFO closure, and the results of the MIST trial, which has a decrease by $3.26 \pm 1.82$ days of headache attacks in the PFO closure group after 6-month follow-up. ${ }^{9}$ The investigators of the present study also noted that headache frequency, headache duration, HIT-6 score, and VAS score all improved following PFO closure.

A retrospective analysis involving 54 studies was published in $2016,{ }^{8}$ of which 20 studies included 2444 patients with migraine, and the incidence of PFO ranged from $15 \%$ to 90\%, including MA (16\%-45\%) and MoA (11\%-34\%). In the present study, there were $43(32.09 \%)$ patients with MA with PFO. The HIT-6 score was higher in MA than that in MoA before PFO closure $(p=0.009)$. However, there was no significant difference in efficacy in PFO closure between patients with MA and patients with MoA $(\mathrm{p}>0.05)$. With the development of diagnostic modalities, the identification rate of PFO, ASA, and ASD has been significantly increased in patients with migraine. In our study, all 134 patients were identified with large PFO by cTTE. According to the TTE results in the resting state, 134 patients presented with shunt of different sizes. Merely the VAS score significantly improved in patients with small shunts at 3 months after PFO closure $(p=0.036)$. Moreover, 10 patients had ASA, 4 patients had ASD, and 1 patient had coexisting ASA and ASD. Nevertheless, the investigators in the present study did not find a significant difference in headache frequency, headache duration, VAS score, or HIT-6 score among patients with $\mathrm{PFO}$ of different phenotypes. At present, there is little evidence on the correlation between ASA/ASD and migraine. A previous study revealed that the incidence of a further event was higher in patients with PFO and concomitant ASA after cryptogenic stroke. ${ }^{23}$ It is possible that ASA induces platelet aggregation and vasoactive substances, or platelets may pass through the foramen ovale. ${ }^{24}$

Above all, our single-center study found that PFO closure can effectively reduce headache symptoms and improve quality of life. We try to identify possible causes of differences between RCTs and single-center studies. First, all patients in MIST were patients with MA. PREMIUM had around $65 \%$ of the subjects in the closure group who had aura, which is significantly higher than the general prevalence of migraine with aura. PRIMA and PREMIUM also had limitations with slow recruitment/high screening:recruitment ratio. The authors of MIST noted that two patients in the closure arm were responsible for $20 \%$ of all headache days during the analysis period, and only with exclusion of these two patients would there be a significant reduction in migraine days between the two groups. Thus, the inclusion criteria, the primary/secondary endpoints, and follow-up times in the three RCTs were inconsistent and the control group may have placebo effects on patients. Short follow-up time may also be one of the reasons for the poor efficacy of PFO closure. However, there seems to be no such strict regulation in single-center studies.

Then, three RCTs may underestimate the impact of residual shunt, which may still cause headache attacks. Due to the likely presence of pulmonary shunts, right-toleft shunting cannot be eliminated $100 \%$ by PFO closure. Some scholars have suggested that "Paradoxical Embolism" event may still occur in non-endothelialized areas, which may cause headache attacks. ${ }^{2325} 26$ A study published in the Journal of Cardiovascular Intervention on February 10, 2020 showed that migraine burden was reduced by $>50 \%$ in $87.0 \%$ of patients, and symptoms were completely abolished in 48\%. At 6 months after PFO closure, 26\% patients had residual right-to-left shunt. Absence of right-to-left shunt was associated with improvement in migraine burden by $>50 \%($ OR $4.60 ; 95 \%$ CI 1.30 to $16.10 ; \mathrm{p}=0.017) .{ }^{27}$ As our findings, 87 patients were re-examined by cTTE at 6 months, in which $31.03 \%$ patients presented residual shunt.

Furthermore, all patients had taken antiplatelet aggregation drugs after PFO closure, such as aspirin and clopidogrel. Aspirin, as a non-steroidal anti-inflammatory drug, is one of the medications for migraine prevention. Besides, the conclusion of our another research presented as a poster in International Headache Society 2019 provided some evidence that clopidogrel $75 \mathrm{mg} /$ day could act as an effective complementary prophylactic for migraine with PFO in patients who have poorly responded to routine prophylactics. Therefore, we surmised that antiplatelet agglutination therapy may be the main reason for the difference between PFO closure and sham group.

In addition, some studies used different screening methods and definitions of right-to-left shunts. For instance, the cut-point for a significant PFO was variously set as 10 bubbles, ${ }^{28} 20$ bubbles, ${ }^{29-32} 25$ or 30 bubbles, ${ }^{933}$ or occasionally, 50 bubbles. ${ }^{28}$ This inconsistency may lead to controversial conclusions.

There were some limitations to the present study. First, this was a single-center, small-sample-size, non-randomized trial. Second, there may be a placebo effect after PFO closure. Third, antiplatelet aggregative agents may have played a role in the improvement of migraine. In the future, a larger-scale clinical trial with long-term follow-up will be conducted to assess the efficacy of PFO closure for treating migraine and identify the definitive indications for PFO closure in detail.

\section{CONCLUSION}

This study confirmed that PFO closure can effectively reduce the frequency and duration of migraine and improve 
quality of life. By exploring the differences between RCTs and single-center studies, the definitive indications and long-term effect of PFO closure still need further research.

Acknowledgements The authors thank the participants, investigators, and staff of the First Affiliated Hospital of Xi'an Jiaotong University, who were involved in this study.

Contributors $G L$ and $Y Q$ designed the study, collected the data, and drafted the manuscript. GL revised the manuscript. YZ and GC performed the operations. RL and YD performed the CTTE examinations. XL and HX assisted in the data collection. YC and YG performed the statistical analyses. All authors have read and approved the final manuscript.

Funding This study was supported by the National Key Technology R\&D Program of China (No. 2014BAI04B05).

Competing interests None declared.

Patient consent for publication Not required.

Ethics approval The study was approved by the Ethics Committee of the First Affiliated Hospital of Xi'an Jiaotong University (KYLLSL-2013-007-01).

Provenance and peer review Not commissioned; externally peer reviewed.

Data availability statement Data are availabe upon reasonable request.

Open access This is an open access article distributed in accordance with the Creative Commons Attribution Non Commercial (CC BY-NC 4.0) license, which permits others to distribute, remix, adapt, build upon this work noncommercially, and license their derivative works on different terms, provided the original work is properly cited, an indication of whether changes were made, and the use is non-commercial. See: http://creativecommons.org/ licenses/by-nc/4.0/.

\section{ORCID iDs}

Yi Qi http://orcid.org/0000-0003-3687-735X

Yichen Guo http://orcid.org/0000-0002-0417-9795

\section{REFERENCES}

1 Disease G, Incidence I, Collaborators P, et al. Global, regional, and national incidence, prevalence, and years lived with disability for 310 diseases and injuries, 1990-2015: a systematic analysis for the global burden of disease study 2015. Lancet 2016;388:1545-602.

2 Meissner I, Whisnant JP, Khandheria BK, et al. Prevalence of potential risk factors for stroke assessed by transesophageal echocardiography and carotid ultrasonography: the SPARC study. Stroke Prevention: Assessment of Risk in a Community. Mayo Clin Proc 1999;74:862-9.

3 Ning M, Lo EH, Ning P-C, et al. The brain's heart-therapeutic opportunities for patent foramen ovale (PFO) and neurovascular disease. Pharmacol Ther 2013;139:111-23.

4 Del Sette $M$, Angeli $\mathrm{S}$, Leandri $\mathrm{M}$, et al. Migraine with aura and right-toleft shunt on transcranial Doppler: a case-control study. Cerebrovasc Dis 1998:8:327-30.

5 Schwedt TJ, Demaerschalk BM, Dodick DW. Patent foramen ovale and migraine: a quantitative systematic review. Cephalalgia 2008;28:531-40.

6 Anzola GP, Magoni M, Guindani M, et al. Potential source of cerebral embolism in migraine with aura: a transcranial Doppler study. Neurology 1999;52:1622-5.

7 Wilmshurst P, Nightingale S. Relationship between migraine and cardiac and pulmonary right-to-left shunts. Clin Sci 2001;100:215-20.

8 Tariq N, Tepper SJ, Kriegler JS, et al. Patent foramen ovale and migraine: closing the debate-a review. Headache 2016;56:462-78.

9 Dowson A, Mullen MJ, Peatfield R, et al. Migraine Intervention With STARFlex Technology (MIST) trial: a prospective, multicenter, double-blind, shamcontrolled trial to evaluate the effectiveness of patent foramen ovale closure with STARFlex septal repair implant to resolve refractory migraine headache. Circulation 2008;117:1397-404.
10 Mattle HP, Evers S, Hildick-Smith D, et al. Percutaneous closure of patent foramen ovale in migraine with aura, a randomized controlled trial. Eur Heart $J$ 2016;37:2029-36

11 Tobis JM, Charles A, Silberstein SD, et al. Percutaneous closure of patent foramen ovale in patients with migraine: the premium trial. J Am Coll Cardiol 2017;70:2766-74

12 Ha JW, Shin MS, Kang S, et al. Enhanced detection of right-to-left shunt through patent foramen ovale by transthoracic contrast echocardiography using harmonic imaging. Am J Cardiol 2001;87:669-71.

13 Post MC, Budts W. The relationship between migraine and right-to-left shunt: fact or fiction? Chest 2006;130:896-901.

14 Tepper SJ, Cleves C, Taylor FR. Patent foramen ovale and migraine: association, causation, and implications of clinical trials. Curr Pain Headache Rep 2009;13:221-6.

15 Anzola GP, Mazzucco $S$. The patent foramen ovale-migraine connection: a new perspective to demonstrate a causal relation. Neurol Sci 2008;29:15-18.

16 Schwerzmann M, Nedeltchev K, Lagger F, et al. Prevalence and size of directly detected patent foramen ovale in migraine with aura. Neurology 2005:65:1415-8.

17 Dalla Volta G, Guindani M, Zavarise P, et al. Prevalence of patent foramen ovale in a large series of patients with migraine with aura, migraine without aura and cluster headache, and relationship with clinical phenotype. J Headache Pain 2005;6:328-30.

18 Nozari A, Dilekoz E, Sukhotinsky I, et al. Microemboli may link spreading depression, migraine aura, and patent foramen ovale. Ann Neurol 2010:67:221-9.

19 Morandi E, Anzola GP, Angeli S, et al. Transcatheter closure of patent foramen ovale: a new migraine treatment? J Interv Cardiol 2003;16:39-42.

20 Slavin L, Tobis JM, Rangarajan K, et al. Five-year experience with percutaneous closure of patent foramen ovale. Am J Cardiol 2007;99:1316-20.

21 Dubiel M, Bruch L, Schmehl I, et al. Migraine headache relief after percutaneous transcatheter closure of interatrial communications. J Interv Cardiol 2008;21:32-7.

22 Mas JL, Arquizan C, Lamy C, et al. Recurrent cerebrovascular events associated with patent foramen ovale, atrial septal aneurysm, or both. $N$ Engl I Med 2001;345:1740-6.

23 Chambers J, Seed PT, Ridsdale L. Association of migraine aura with patent foramen ovale and atrial septal aneurysms. Int J Cardiol 2013;168:3949-53.

24 Cabanes L, Coste J, Derumeaux G, et al. Interobserver and intraobserver variability in detection of patent foramen ovale and atrial septal aneurysm with transesophageal echocardiography. J Am Soc Echocardiogr 2002;15:441-6.

25 Diener $\mathrm{H}-\mathrm{C}$, Schorn CF, Bingel U, et al. The importance of placebo in headache research. Cephalalgia 2008;28:1003-11.

26 Schwerzmann M, Meier B. Impact of percutaneous patent foramen ovale closure on migraine course. Interv Cardiol 2010;2:177-87.

27 Ben-Assa E, Rengifo-Moreno P, Al-Bawardy R, et al. Effect of residual interatrial shunt on migraine burden after transcatheter closure of patent foramen ovale. JACC Cardiovasc Interv 2020;13:293-302.

28 Daniëls C, Weytjens C, Cosyns B, et al. Second harmonic transthoracic echocardiography: the new reference screening method for the detection of patent foramen ovale. Eur J Echocardiogr 2004;5:449-52.

29 Braun MU, Fassbender D, Schoen SP, et al. Transcatheter closure of patent foramen ovale in patients with cerebral ischemia. J Am Coll Cardiol 2002:39:2019-25

30 Wahl A, Praz F, Tai T, et al. Improvement of migraine headaches after percutaneous closure of patent foramen ovale for secondary prevention of paradoxical embolism. Heart 2010;96:967-73.

31 Wilmshurst PT, Nightingale S, Walsh KP, et al. Clopidogrel reduces migraine with aura after transcatheter closure of persistent foramen ovale and atrial septal defects. Heart 2005;91:1173-5.

32 Garg P, Servoss SJ, Wu JC, et al. Lack of association between migraine headache and patent foramen ovale: results of a case-control study. Circulation 2010;121:1406-12.

33 Sztajzel R, Genoud D, Roth S, et al. Patent foramen ovale, a possible cause of symptomatic migraine: a study of 74 patients with acute ischemic stroke. Cerebrovasc Dis 2002;13:102-6. 